\title{
Non-Invasive Assessment of Sentinel Lymph Nodes That Drain the Tumoral Mammary Glands in Female Dog
}

\author{
Florin Gheorghe STAN
}

Faculty of Veterinary Medicine, University of Agricultural Sciences and Veterinary Medicine, Calea Mănăștur, 3-5, Cluj Napoca, Romania

*corresponding author: florin.stan@usamvcluj.ro

Bulletin UASVM Veterinary Medicine 73(2) / 2016,

Print ISSN 1843-5270; Electronic ISSN 1843-5378

DOI:10.15835/buasvmcn-vm: 12251

\begin{abstract}
Mammary gland tumours occupy a significant place in the pathology of this species. Female dogs that are not spayed after their first heat cycle have a higher predisposition of developing mammary tumours. Most tumours metastasize at distance via the lymphatic system. In these conditions, the sentinel lymph nodes of the mammary glands must be assessed prior to surgical treatment.

Considering the insufficient usage of non-invasive investigative methods of the sentinel lymph nodes, the aim of this study is to describe the sonographic anatomy of the lymph nodes that drain the mammary gland tumours in female dog.

Twelve dog females presenting tumours of the cranial and caudal abdominal mammary glands (A1 and A2), inguinal mammary gland (I) and cranial thoracic mammary gland (T1) were examined (group I). In addition, a control group composed of eight dog females was used (group II). The axillary and superficial inguinal lymph nodes were evaluated using an algorithm composed of gray-scale ultrasound, Doppler technique, contrast enhanced ultrasound (CEUS) and real time elastography. Surgical excision of the sentinel lymph nodes was performed and samples for histopathological examination were taken.

The following ultrasonographic findings revealed on gray-scale examination were suspected for the metastatic infiltration: hypoechoic pattern, round shape, hillus absence and heterogenicity. Doppler technique showed an aberrant and mixed vascularisation of the lymph nodes, while the CEUS revealed incomplete enhancement of lymph nodes parenchyma. On real time elastography, the presence of blue areas in more than $50 \%$ from the lymph nodes parenchyma led us to conclude that the lymph node stiffness was caused by metastatic infiltration. Histopathological examination confirmed the presence of the metastatic infiltration in $97 \%$ of the examined lymph nodes.

The algorithm composed of gray-scale ultrasound, Doppler technique, CEUS and real time elastography proved to be efficient in diagnosing the metastatic infiltration of sentinel lymph nodes of mammary gland. Further studies are needed to validate the proposed algorithm.
\end{abstract}

Keywords ultrasonography, lymph nodes, female dog, mammary gland tumour

\section{INTRODUCTION}

Neoplasia is a global reality, which is rapidly growing in many countries, both in human and veterinary medicine. In female dog, there is an increasing incidence of mammary gland tumour, one of four unsprayed female developing malignant disease (Cassali et al.,2014; Stan et al., 2010). It is also important to note that a high number of practitioners, have reported in recent years the diagnosis of malignant melanomas and 
lymphomas in veterinary medicine (Goldberg et al., 2004, 2011), especially in pets. The status of regional lymph nodes is a well-known prognosis factor in most malignant tumours in both humans and animals, holding a major impact in choosing the therapy (Nyman et al., 2005; Servais et al., 2011).

Pre-therapeutic staging includes assessment of the extent of the neoplasm through the lymphatic system. The most important lymph node in assessing lymphatic dissemination is the so-called "sentinel lymph node" (Sever et al., 2012; Stan et al., 2010; 2012). Regarding the link between mammary tumour and regional lymph nodes, the reaction of potential lymph node, is related to tumour topography and the direction of lymphatic drainage (Stan et al., 2012; 2013; 2014).

In recent years, non-invasiveness has become a major goal in malignant diseases, since the imaging methods are continuously developing (Alam et al., 2008; Liu et al., 2014; Rubaltelli et al., 2014; Stan et al., 2014). However, lymph node biopsy is currently considered the main procedure to investigate the status of lymph nodes (Sever et al., 2012). Considering that the accuracy of biopsy is only $70-85 \%$, depending on the size of the fragments collected, and it is influenced by errors of sampling and sample processing, there is a tendency of finding an alternate, non-invasive investigating technique for staging malignant disease. The most suitable in this way are the imaging techniques.

This study is fully justified, both from theoretical (establishing the criteria for noninvasive investigation of lymph nodes) and practical considerations (compiling an ultrasonographic diagnostic algorithm), due to many similarities (hormonal status, estrogens-dependent breast tumours) between the lymphatic drainage of the mammary gland (Cassali et al., 2014; Stan et al., 2012) and also the variability of this system in both humans and carnivores.

\section{MATERIALS AND METHODS}

The draining lymph nodes of mammary glands belonging to the twelve dog females presenting tumours of the cranial and caudal abdominal mammary glands ( $\mathrm{A} 1$ and $\mathrm{A} 2, \mathrm{n}=4$ ), inguinal mammary gland $(I, n=6)$ and cranial thoracic mammary gland ( $\mathrm{T} 1, \mathrm{n}=2)$ were examined. The selection criteria are listed below:

$$
\begin{array}{c|c}
\text { Inclusion criteria } & \begin{array}{c}
\text { Exclusion criteria } \\
\text { - Subjects with } \\
\text { mammary gland } \\
\text { tumours confirmed } \\
\text { by clinical, } \\
\text { imaging and } \\
\text { histopathological } \\
\text { examinations } \\
\text { Stable general } \\
\text { conditions at } \\
\text { surgically treated } \\
\text { tumours } \\
\text { study beginning } \\
\text { Owner consent }
\end{array} \text { Pregnancy } \\
\end{array}
$$

\section{Exclusion criteria}

In addition, eight healthy subjects were used as control group. All subjects underwent a clinical examination to highlight the potential associated comorbidities. The axillary and superficial inguinal lymph nodes were evaluated using an algorithm composed of gray-scale ultrasound, Doppler ultrasound, contrast-enhanced ultrasound (CEUS) and real-time elastography.

Gray-scale ultrasound of superficial lymph nodes was performed with Logiq $9 \AA$ (General Electric) using a multifrequency linear probe (8$12 \mathrm{MHz}$ ). Doppler, elastographic and contrast enhanced ultrasound examination modes were available, in either real time or post processing. The following characteristics of the lymph nodes have been assessed: shape, size, (transverse and longitudinal axes), capsule delimitation from adjacent tissues, lymph node parenchyma, ecostructure and ecogenicity and hilum ecogenicity.

Doppler ultrasound pursued the following: the presence of vascular signal in lymph nodes, vascular type (normal vs. altered) and vascular pattern (peripheral vs. central).

For CEUS a second-generation contrast agent (SonoVue - Barraco-Italy) was used in two steps:

1. The suspension was prepared in its original packing. A quantity of $0.4 \mathrm{ml}$ SonoVue was injected in the peritumoral area in four distinct points, followed by gentle massage of the injected area. The progression of the contrast agent was followed on the device's screen up the first lymph node, noting the anatomic region where it was found. Also, the time of progression was recorded and the parenchyma's aspect has been assessed.

2. After placing a peripheral venous catheter, an average dose of $0.6 \mathrm{ml}$ SonoVue/animal (0.6mlSonoVue/10kg BW) from the freshly 
prepared suspension was administrated on the main route of the catheter. On the secondary route, $5 \mathrm{ml}$ of physiological serum was injected. When the contrast injection was done, the ultrasound timer started and the pattern of the lymph node parenchyma in the arterial and venous phases was recorded.

Real-time ultrasound elastography evaluated the region of interest (ROI) around the lymph nodes. The colour-coded signals used for the evaluation of the stiffness of tissues (bluevery hard; red-very soft; yellow-soft; greenintermediate) were registered. This data was stored as digital picture files, and subsequently analyzed by using Adobe Photoshop ${ }^{\circledR}$ to estimate the amount of each colour in the ROI numerically.

In all subjects, a sample for histopathological examination was taken. Lymph node ultrasound guided biopsy was performed in healthy subjects. Surgical excision of the sentinel lymph nodes during the mastectomy in subjects presenting mammary tumours was performed and samples for histological examination were taken. Haematoxylin\&Eosin staining was used.

\section{RESULTS AND DISCUSSION}

Forty-eight lymph nodes were evaluated. There have been recorded thirty- two lymph nodes in the malignant category, sixteen in the benign category.

Gray-scale ultrasound. Lymph node patterns were described according to Table 1, which shows the chosen parameters and corresponding scores for determining the lymph node pattern (malignant or benign). The gray-scale ultrasound characteristics used to differentiate between benign and malignant lymph nodes are illustrated in figure 1.

Doppler ultrasound used the following parameters: the presence of vascular signal in the lymph node, vascular type (normal vs altered), vascular pattern (central vs peripheral).

Tab. 1. Gray-scale ultrasound

\begin{tabular}{ccc}
\hline \multicolumn{1}{c}{ Parameter } & \multicolumn{2}{c}{ Characteristic } \\
\hline S/L* ratio & Benign & Malignant \\
\cline { 2 - 3 } Echogenicity & $<0.5$ & $>0.5$ \\
Echostructure & Isoechoic/Hyperechoic & Hypoechoic \\
Capsule features & Homogenous & Inhomogeneous \\
Hilum & Well defined with regular margins & Irregular \\
Nr. of points & Present, hyperechoic & Absent \\
\hline${ }^{* S}=$ short axis, L $=$ long axis & 0 & 1 \\
\hline
\end{tabular}
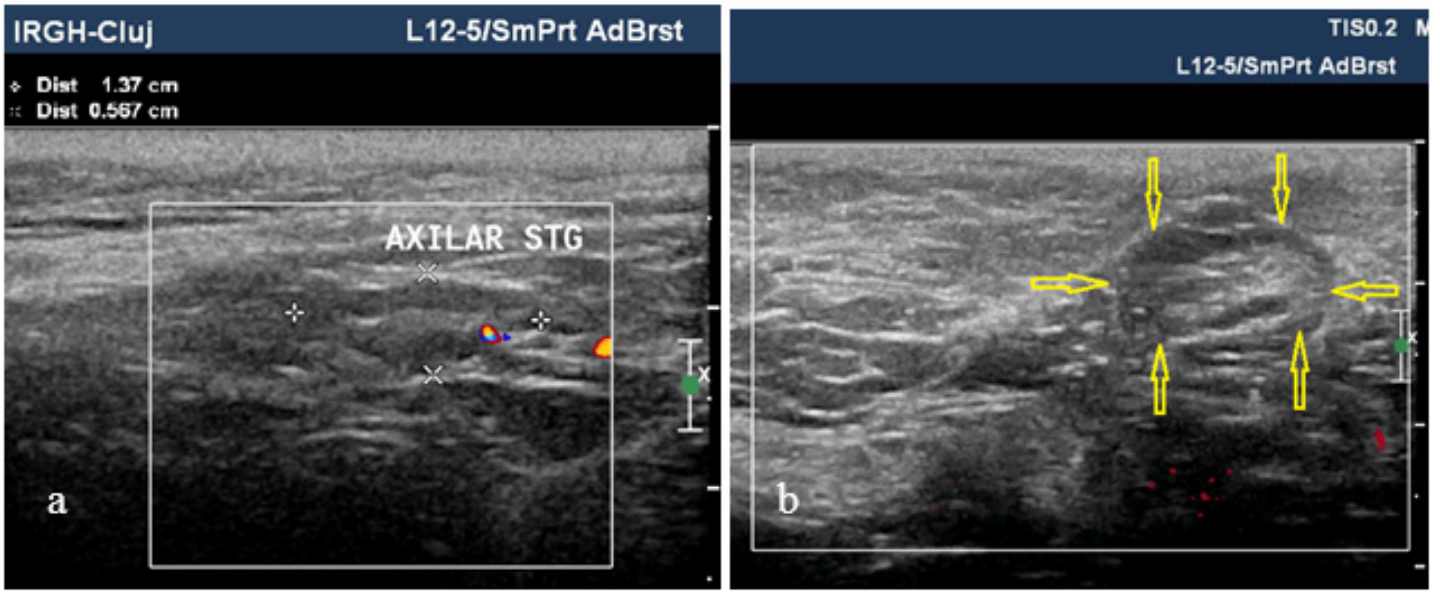

Fig. 1. Grey scale US of normal (a) and pathological (b) lymph node. a) Oval shape, well defined capsule and homogenous echostructure of normal lymph node; b) Rounded shape, irregular appearance of capsule and inhomogeneous echostructure of pathological lymph node - arrows. 
Each pattern has a score specified in Table 2 and illustrated in figure 2 .

Using contrast enhanced ultrasound (CEUS) two patterns of enhancement in sentinel lymph nodes were observed: complete enhancement and partial enhancement with the presence of perfusion defects. Contrast-enhanced ultrasound performed after peritumoral administration of 0.4 $\mathrm{ml}$ SonoVue in four points revealed the presence of hypoperfused focal areas of sentinel lymph nodes in eleven subjects from group I. After intravenous administration of contrast agent, the pattern of enhancement was analyzed in both groups. The results and score for each accomplished parameter are detailed in Table 3. The two pattern of enhancement are illustrated in figure 3.

on real time elastography, the colour-coded signals (blue-very hard, red-very soft, yellow-soft

Tab. 2. Color Doppler

\begin{tabular}{ccc}
\hline \multicolumn{2}{c}{ Parameter } & Characteristic \\
\cline { 2 - 3 } & Penign & Malignant \\
\cline { 2 - 3 } $\begin{array}{c}\text { Presence of hilar } \\
\text { vascular signal } \\
\begin{array}{c}\text { Pattern } \\
\text { Distribution }\end{array}\end{array}$ & $\begin{array}{c}\text { Ordered } \\
\text { Radially from the hilum } \\
\text { toward the capsule }\end{array}$ & Absent \\
Nr. of points & 0 & $\begin{array}{c}\text { Chaotic/Unordered } \\
\text { Peripheral, subcapsular }\end{array}$ \\
\hline
\end{tabular}
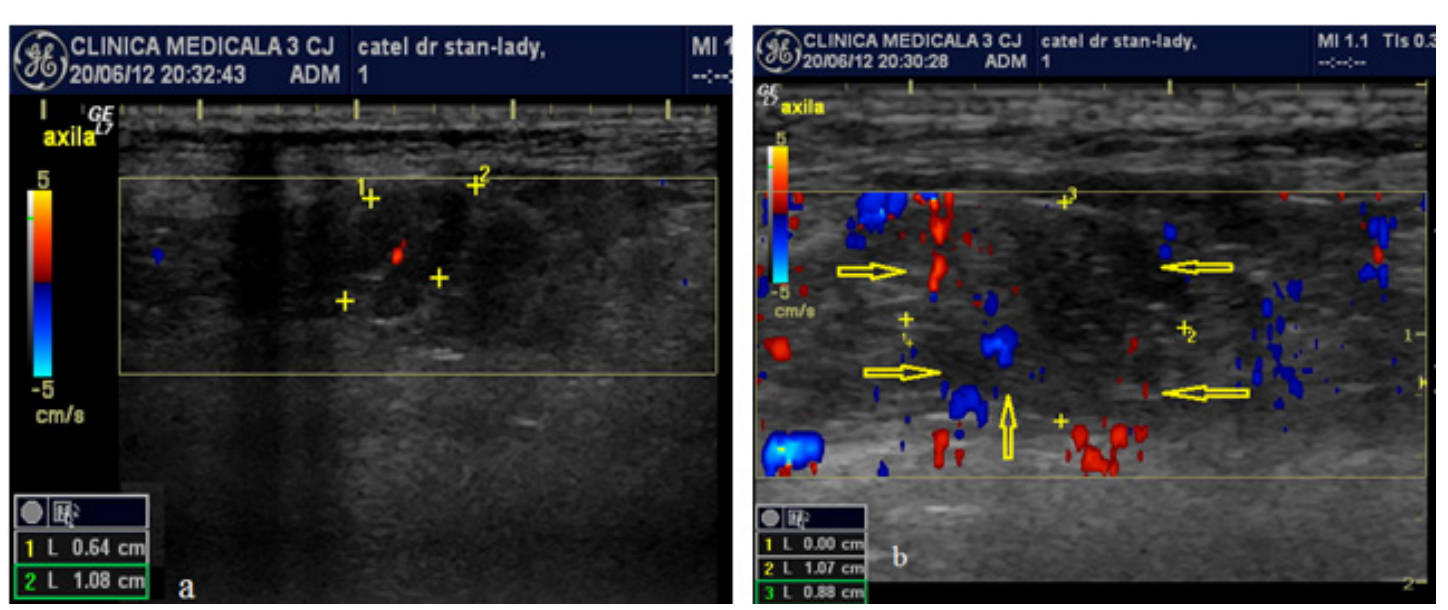

Fig. 2. Doppler US of normal (a) and pathological (b) lymph node. a) The presence of hilar vascular signal in axillary lymph node. b) Chaotic arrangement with peripheral distribution of the vessels in a metastatic lymph node.

Tab. 3. CEUS

\begin{tabular}{ccc}
\hline & Parameter & Characteristic \\
\cline { 2 - 3 } a) peritumoral adm & Benign & Malignant \\
Enhancement & Complete, intensive & Incomplete, with areas without contrast agent \\
Type & Homogenous & Inhomogeneous \\
Distribution & Centripetal & Aberrant \\
b) IV adm. & Complete, intensive & Incomplete with filling defects or absent \\
Enhancement & Homogenous & Inhomogeneous \\
Type & Centrifugal & Aberrant, distribution delays \\
Distribution & 0 & 1 \\
\hline Nr. of points & &
\end{tabular}


green-intermediate) were interpretated according to the Table 4.

The result of the gray scale ultrasound and Doppler examination were interpretated as follows:

Number of points less than 4: the nodes were interpretated as benign - ( $\mathrm{n}=16$ lymph nodes).

Number of points ranged between 4 and 6 : the nodes were interpreted as uncertain followed by CEUS and real-time elastography $(\mathrm{n}=10 \mathrm{lymph}$ nodes).

Number of points more than 6: the nodes were considered malignant ( $\mathrm{n}=32$ lymph nodes).

Nowadays the ultrasound exam is the method of first choice in the evaluation of adenopathies by conventional techniques, namely the grey-scale ultrasound and Doppler techniques (Stan 2010; Stan et al., 2012), along with CEUS - ContrastEnhanced Ultrasound (Stan and Badea 2012). In these conditions, the ultrasound techniques are the diagnostic methods whose values were most often studied. These could indicate a change in the researcher's interest to use the non invasive techniques in lymph node assessment instead of conventional invasive methods. Gray scale ultrasound exam studies the diagnostic value of lymph node parenchyma ecogenicity, ecostructure, short to long axis ratio, hilar pattern and margins in both humans and animals (Dudea et al., 2012; Whitman et al., 2011). According to existing literature the two-dimensional ultrasound exam cannot make a real distinction between tumour-infiltrated lymph nodes (malignant ones) and inflammatory lymph nodes - which can be considered as beningn lymph nodes (Dudea et al., 2012; Nyman et al., 2005; Stan et al., 2010). The performances improved by using Doppler ultrasound in evaluating the lymph vascularisation, but not enough (Furukawa et al., 2007; Stan et al., 2010, 2013).

Contrast-enhanced ultrasound (CEUS), has added a new dimension to diagnostic possibilities,
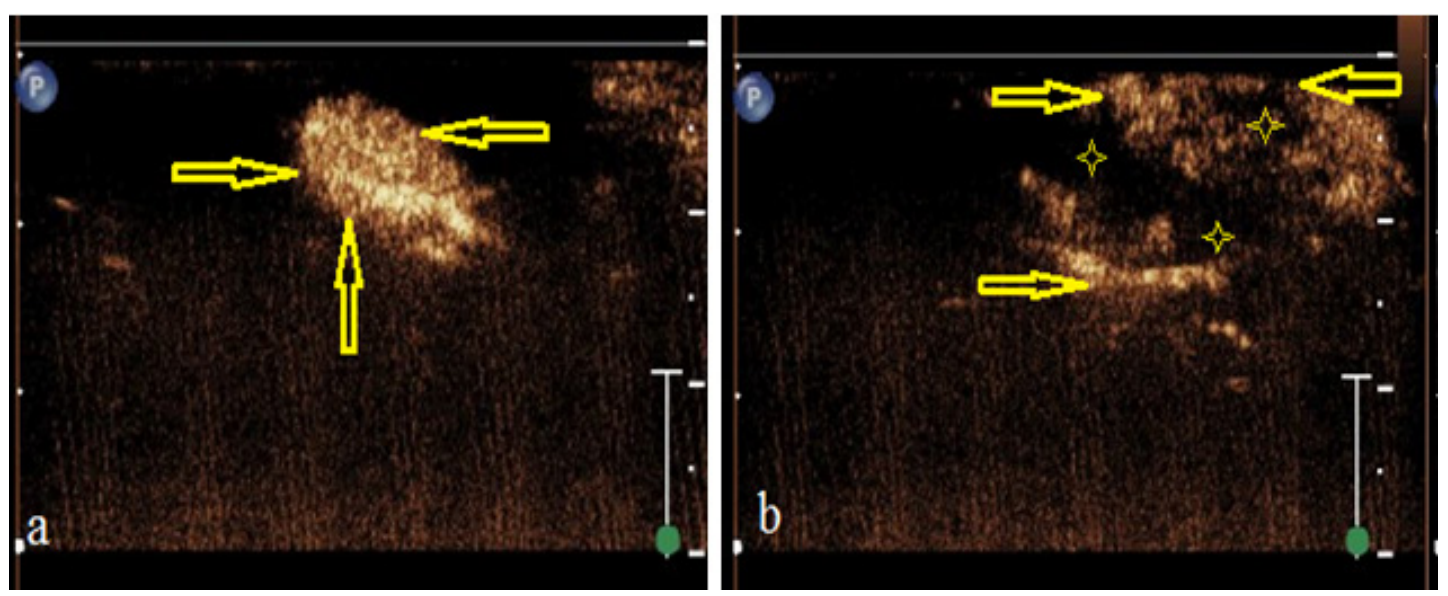

Fig. 3. CEUS of superficial inguinal lymph node. a) Complete, homogenous enhancement in normal lymph node. b) Arterial phase showed several nonenhancing areas within the sentinel lymph nodes of tumoral mammary glands: a hypoperfused mass-asterisks, surrounded by relative normal enhancing of lymph node parenchyma - arrows.

Tab. 4. Real-time elastography

\begin{tabular}{|c|c|c|}
\hline & Parameter & Characteristic \\
\hline & Benign & Malignant \\
\hline $\begin{array}{l}\text { Stiffness, } \\
\text { Quantified by } \\
\text { the software in } \\
\text { colour nuances }\end{array}$ & $\begin{array}{l}\text { a)The whole lymph node is soft, } \\
\text { mostly green, yellow or turquoise } \\
\text { b) }>50 \% \text { of lymph node is green, } \\
\text { with presence of small yellow or } \\
\text { turquoise hyperechogenous nodules } \\
\text { c) }<50 \% \text { of lymph node is blue }\end{array}$ & $\begin{array}{l}>50 \% \text { of lymph node is blue } \\
>50 \% \text { is blue except for some } \\
\text { hyperechogenous nodules } \\
\text { All lymph node is blue and/or shares } \\
\text { the color with surrounding tissue } \\
\text { Hard lymph node colored blue on the } \\
\text { edges, with necrotic areas - green/red }\end{array}$ \\
\hline
\end{tabular}


by establishing an accurate diagnosis of focal lesions and trying to find its utility in assessing lymph nodes status (Goldberg et al., 2004, 2005; 2011). By using the peritumoral injection of Sonaziod in 63 Sinclair swine with melanoma, Goldberg and al., (2011) stated the importance of this method in lymphatic mapping and its ability to localize the sentinel lymph nodes in cases of unpredictable drainage pathways. However, their results have shown that the differentiation between the benign and malignant lymph nodes was limited using only lymphosonography. Analysis of characteristic parameters of the method such as washing curves, transit times and quantifying the amount of contrast, improved the method, especially in describing the free lymph nodes (Stan et al., 2014).

Another imaging technique is ultrasound elastography (real-time elastography), which quantifies the degree of tissue stiffness. Diagnostic methods based on this technique seem to be promising (Alam et al., 2008; Das et al., 2011; Lenghel et al., 2012). In real-time ultrasound elastography, visualising the stiffness of the tissue requires transposition in colour mode, each colour signifying a level of stiffness. Thus, very hard tissues are represented by dark blue and intermediate shades of green, soft tissue are yellow and very soft ones are red. Evaluating this colours by using a special software, certain scores of elasticity can be calculated (Dudea et al., 2012; Lenghel et al., 2012). Based on four or five colorcoded patterns of lymph nodes, an elasticity score was proposed for the metastatic lymph nodes of the head and neck in squamos carcinoma. Alam et al., (2008) established five elastographic patterns of lymph nodes based on the distribution and percentage of hard areas (or high elasticity) in lymph node parenchyma; pattern 1 being an absent or very small hard area to pattern 5 , in which the hard area occupied the entire node. In this study the cut-off line between benign and malignant lymph nodes was set between pattern 2 and 3 . Recently, Lenghel et al., (2012) report a very good specificity and sensitivity in the differentiation between malignant and benign lymph nodes using the combined gray-scale appearance and elastographic images. The elastographic score that also includes the structural changes was based on eight patterns (Lenghel et al 2012). Our study proposed an elasticity score of four patterns, trying to include the structural changes that indicated tumour infiltration too.

Currently, there is no standard of non-invasive lymph node staging in veterinary medicine. The current surgical approach by total excision of the lymph node without a prior investigation is an invasive procedure with potential risk of complications. It presents a high degree of intraoperative subjectivity and even errors in proper identification of the first lymphatic drainage station may occur. We consider that it is time to change the mainstream approach of tumour staging investigation in veterinary medicine with a non-invasive and less expensive alternative.
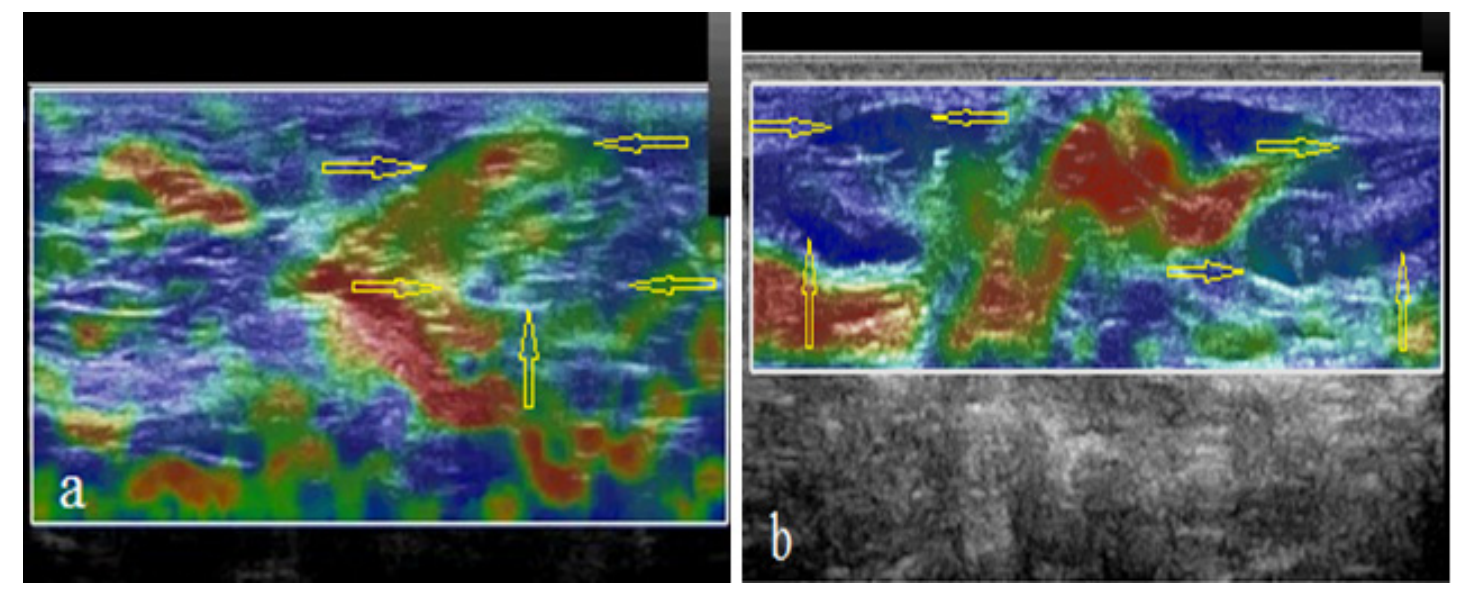

Fig. 4. Real time elastography of sentinel lymph nodes. a) Benign lymph nodes were mostly green; b) Hard lymph nodes revealed by the blue color present in all lymph node (the superficial inguinal lymph nodes) indicate the presence of metastatic infiltration. 


\section{CONCLUSIONS}

The combination of the four ultrasound techniques used in the current study is a premiere. They were represented by: grey-scale ultrasound, Doppler ultrasound, contrast-enhanced ultrasound and real-time elastography. By using this algorithm, the metastatic infiltration of sentinel lymph nodes of mammary gland was assessed. These preliminary results are the first ones reported in veterinary medicine. Unfortunately, the statistical analysis is not relevant due to the low number of clinical cases, this being one of the major limitations of the proposed algorithm. Further studies are needed to confirm the accuracy of the diagnosis and to compare the results to those obtained by CT or MRI.

\section{REFERENCES}

1. Alam F, Naito K, Horiguchi J, Fukuda H, Tachikake T, Ito K. (2008). Accuracy of sonographic elastography in the differential diagnosis of enlarged cervical lymph nodes: comparison with conventional B-mode sonography. AJR Am J Roentgenol. 191:604-610.

2. Cassali $G D$, Lavalle GE, Ferreira E, Lima AE, De Nardi AB, Ghever C, Sobral RA, et al., (2014). Consensus for the Diagnosis, Prognosis and Treatment of Canine Mammary Tumors. Brazilian Journal of Veterinary Pathology 7(2):38 - 69.

3. Das D, Gupta M, Kaur H, Kalucha A (2011). Elastography: the next step. J Oral Sci;53:137-141.

4. Dudea SM, Lenghel M, Botar-Jid C, Vasilescu D, Duma M (2012). Ultrasonography of superficial lymph nodes: benign malignant. Med Ultrason 14: 294-306.

5. Furukawa MK, Kubota A, Hanamura H, Fujita Y, Furukawa M (2007). Diagnosis of cervical lymph node metastasis of head and neck squamous cell carcinoma: usefulness of power Doppler ultrasonography and elastography. MEDIX (Suppl) :20-23.

6. Goldberg BB, Merton DA, Liu JB, (2004).Sentinel lymph nodes in a swine model with melanoma: contrastenhanced lymphatic US. Radiology 230:727-734.

7. Goldberg BB, Merton DA, Liu JB, Murphy G, Forsberg F. (2005). Contrast-enhanced sonographic imaging of lymphatic channels and sentinel lymph nodes. J Ultrasound Med 24:953-965.

8. Goldberg BB, Merton DA, Liu JB, et al (2011). Contrastenhanced ultrasound imaging of sentinel lymph nodes after peritumoral administration of Sonazoid in a melanoma tumor animal model. J Ultrasound Med 30:441-453.

9. Lenghel LM, Bolboaca SD, Botar-Jid C, Baciut G, Dudea SM. (2012) The value of a new score for sonoelastographic differentiation between benign and malignant cervical lymph nodes. Med Ultrason 14:271-277.

10. Liu JB, Merton DA, Berger AC (2014), Contrast-enhanced sonography for detection of secondary lymph nodes in a melanoma tumor animal model. J Ultrasound Med 33: 939-947.
11. Nyman, H.T., Kristensen, A.T., Skovgaard, I.M (2005) Characterization of normal and abnormal canine superficial lymph nodes using grey-scale B-mode, color flow mapping, power and spectral Doppler ultrasonography a multivariate study. Vet Radiol Ultrasound 46: 404-410

12. Rubaltelli L, Beltrame V, Scagliori E. (2014). Potential use of contrast-enhanced ultrasound (CEUS) in the detection of metastatic superficial lymph nodes in melanoma patients. Ultraschall Med 35: 67-71.

13. Servais EL, Colovos C, Bograd AJ, White J, Sadelain M, Adusumilli PS (2011), Animal models and molecular imaging tools to investigate lymph node metastases. J Mol Med (Berl) 89:753-769.

14. Sever, AR, Mills P, Weeks J, Jones S, Fish D, Jones P A, Mali W (2012). Preoperative needle biopsy of sentinel lymph nodes using intradermal microbubbles and contrast-enhanced ultrasound in patients with breast cancer. American Journal of Roentgenology 199(2):465470.

15. Stan F, Gudea A, Baba AI, Feier D, Badea R (2012). Correlation between Ultrasonographic Features and Morphological Pattern after Blue Dye Injection of Normal Superficial Lymph Nodes in Carnivores. Bulletin UASMV Cluj Napoca 69 (1-2):211 - 219

16. Sta, F (2010). Power Dopler ultrasonography vs Color Dopler of the Sentinel Lymph Mammary Glands at Female Dog. Bulletin of University of Agricultural Sciences and Veterinary Medicine Cluj-Napoca. Veterinary Medicine 67 (1): 298-304.

17. Stan F, Damian A, Papuc I, Gudea A, Chirilean I, Dezdrobitu C, Bochis I, Crisan M, Abu Rock Ayman, Irimescu I, Tuns F (2010). The Importance of Ultrasonography in the Exploration of the Superficial Lymphatic System of Carnivores, Bulletin of University Agricultural Sciences and Veterinary Medicine Cluj-Napoca, Veterinary Medicine 67(1): 291-297.

18. Stan F, Badea R (2012). Morphological Identification of Lymph Node Vasculature Using Contrast Enhanced Ultrasonogaphy (CEUS). Bulletin of University of Agricultural Sciences and Veterinary Medicine ClujNapoca. Veterinary Medicine 69(1-2):197-203.

19. Stan F, Damian A, Gudea A, Dezdrobitu C, Delia Bob, Martonoş C, Lăcătuş R, Purdoiu R, Papuc I, Bochis I (2013). Normal anatomy of popliteal lymph center, anatomical variants and non invasive assesment using ultrasound techniques. Lucrări Științifice, Seria Medicină Veterinară, Iași 55(3-4): 373-380.

20. Stan F, Gudea A, Damian A, Papuc I, Bochis I, Pentea M (2014). Grey Scale Ultrasound, CEUS and Elastography to Asses Sentinel Lymph Nodes in Mammary Gland Neoplasia in Carnivores. Anatomia, Histologia, Embryologia, 43: Issue Supplement S1: 88.

21. Whitman GJ, Lu TJ, Adejolu M, Krishnamurthy S, Sheppard D (2011). Lymph node sonography. Ultrasound Clinics 6(3): 369-380.

The Institutional Bioethics Committee of University of Agricultural Science and Veterinary Medicine in accordance to Directive 2010/63 /EU of the European Parliament and of the Council on the protection of animals used for scientific purposes approved the study. 